\title{
Analyse multi-niveaux des freins à l'adoption de la certification du cacao au Cameroun
}

\author{
Anny Lucrèce NLEND NKOTT • Innovation, Université de Montpellier, CIRAD, INRAE, Montpellier \\ SupAgro, Montpellier, France \\ CIRAD, UMR Innovation, F-34398 Montpellier, France \\ annylucrecenlendnkott@yahoo.com \\ Syndhia MATHÉ • Innovation, Université de Montpellier, CIRAD, INRAE, Montpellier SupAgro, \\ Montpellier, France \\ CIRAD, UMR Innovation, F-34398 Montpellier, France ; IITA Cameroun, Yaoundé, Cameroun \\ syndhia.mathe@cirad.fr \\ Ludovic TEMPLE • Innovation, Université de Montpellier, CIRAD, INRAE, Montpellier SupAgro, \\ Montpellier, France \\ CIRAD, UMR Innovation, F-34398 Montpellier, France \\ ludovic.temple@cirad.fr
}

Les exigences européennes sur la qualité du cacao conduisent au développement de la certification, pour améliorer la production et la gestion post-récolte des fèves. Cependant, la certification reste peu développée au Cameroun. Cet article mobilise le modèle de la transition sociotechnique pour analyser les déterminants d'adoption de la certification. Des entretiens auprès de 11 acteurs de la filière cacao ont été menés, ainsi qu'une enquête auprès de 90 producteurs. Les résultats mettent en exergue l'incomplétude du système acteur de la certification, qui se traduit par sa faible diffusion. Cette tendance est accentuée par l'absence d'un différentiel de prix aux producteurs entre cacao certifié et cacao ordinaire. La conclusion interroge la capacité des normes de certification à transformer le secteur cacao.

MOTS-CLÉS : cacao, certification, adoption, Cameroun

\section{Multi-level analysis of the barriers to the adoption of cocoa certification in Cameroon}

The European requirements on cocoa quality have led to the development of certification in order to improve the production and post-harvest management of cocoa beans. However, in Cameroon, the average national percentage of certified cocoa is low compared to the overall production. This article uses the sociotechnical transition model to analyze the determining factors of certification adoption. The research is based on interviews with eleven actors in the cocoa sector and a survey of ninety producers. The results highlight the lack of a structured certification system, leading to its low diffusion. Moreover, the similarity between the prices of certified and conventional cocoa aggravates the problem. We conclude with a consideration of whether certification rules will be able to instigate a transformation of the cocoa sector. (JEL: 033)

KEYWORDS: cocoa, certification, adoption, Cameroon

A u Cameroun, le cacao est la principale culture d'exportation et la principale source de revenu monétaire pour $50 \%$ de la population agricole qui cultive environ 420000 hectares de surface arable (KIT Royal Tropical Institute et al., 2010). Près de $90 \%$ de la production est exportée vers l'Europe, comme matière première pour les chocolateries (Hinzen et al., 2010) le reste étant transformé localement.
Depuis les années 1990, l'évolution des attentes sociétales et des normes environnementales conduisent les agroindustries européennes, telles que Mars et Barry Callebault, à se fixer pour objectifs de n'acheter que du cacao certifié d'ici 2020. Ces exigences se sont traduites en 2013 par le rejet de 2000 tonnes de cacao camerounais des ports européens, à cause d'une contamination en hydrocarbures 
aromatiques polycycliques attribuée en partie au séchage des fèves sur le goudron (Bagal et al., 2013). Elles conduisent le programme de relance du cacao et du café du Cameroun à prévoir un ensemble de mécanismes institutionnels pour lier l'accroissement de la productivité et l'amélioration de la qualité environnementale du cacao. Parallèlement à cette initiative s'organisent des activités autour de la certification. Les deux types d'initiatives promeuvent la transformation des systèmes de production et des pratiques post-récolte. La certification porte toutefois un positionnement plus marqué pour la préservation de l'environnement et l'interdiction du travail des enfants. Elle devient ainsi un levier potentiel pour orienter les trajectoires technologiques vers un cacao durable et de meilleure qualité. Pourtant, la production en cacao certifié ne représentait que $3 \%$ de la production totale de cacao camerounais en 2016 (Ngoucheme et al., 2016). Cela interpelle la connaissance des dysfonctionnements du système de certification UTZ qui est le plus répandu et a été introduit au Cameroun en 2010 (Potts et al., 2014).

En mobilisant un référentiel en économie de l'innovation, nous considérons l'émergence et le développement des normes de certification comme des innovations institutionnelles. Nous utilisons le modèle de la transition sociotechnique (Geels et Schot, 2007) afin de situer la nature incrémentale ou radicale de la certification, mettre en exergue sa trajectoire d'évolution et les facteurs explicatifs à sa faible adoption au Cameroun. L'hypothèse posée étant que la certification est une innovation incrémentale qui repose sur un noyau restreint d'acteurs du secteur privé, ce qui limite son adoption. Le test de cette hypothèse sur le plan méthodologique conduit à caractériser le système d'acteurs qui structure le fonctionnement et les coordinations dans la filière cacao certifié.

Notre contribution est organisée en deux parties principales. La première explicite le cadre conceptuel basé sur le modèle de la transition sociotechnique, et la méthodologie. La seconde présente les résultats qui en découlent suivant les acteurs de la certification et leurs rôles, les entraves à son adoption et ses possibilités d'évolution.

\section{Cadre théorique et méthodologique}

\section{Cadre théorique : une approche par la transition}

Diverses approches sont utilisées pour analyser les déterminants d'adoption des innovations. Certains auteurs se basent sur une analyse par triangulation des entretiens avec les promoteurs et les utilisateurs de l'innovation, afin de déterminer les facteurs socio-économiques d'adoption (Mwangi et Kariuki, 2015 ; Long et al., 2016). La faible diversité du type d'acteurs impliqués dans ces études conduit souvent à orienter les résultats sur un champ limité de facteurs. D'autres auteurs conduisent des régressions logistiques des facteurs d'adoption ou de non-adoption pour les utilisateurs (producteurs) (Mabah Tene et al., 2013 ; McDonald et al., 2016 ; Paustian et Theuvsen, 2017). Malgré leur potentiel de généralisation, ces approches se focalisent sur une conception binaire de l'adoption (Crossan et Apaydin, 2010; Starr, 2014) ou sur le seul producteur, qui est considéré comme un agent individuel réagissant à des stimulus économiques exogènes.

Sans prétendre pallier les limites de ces approches, nous optons pour une compréhension systémique (Temple et al., 2018) de l'innovation en mobilisant le modèle de la transition sociotechnique (Bui et al., 2016 ; Elzen et al., 2012 ; Geels et Schot, 2007 ; Ingram, 2018). Ce modèle utilise une représentation multi-niveaux (micro, méso, et macro) et donc multi-acteurs (promoteurs de l'innovation, utilisateurs, chercheurs/universitaires, politiciens, bailleurs de fonds), pour déterminer les facteurs (sociaux, économiques, politiques, 
culturels, etc.) qui inhibent ou facilitent l'adoption d'une innovation. Il permet de comprendre l'émergence de changements institutionnels (dans une situation ex post), ou de les prévoir (dans une situation ex ante). La plupart des études sur la transition sociotechnique visent toutefois à expliquer ex post les mécanismes des transitions. Dans notre cas, nous proposons d'utiliser ce modèle comme outil d'aide à la décision pour orienter les trajectoires de développement d'une innovation institutionnelle, en identifiant les facteurs qui entravent son adoption.

Le modèle repose sur la différenciation de trois niveaux que sont la niche d'innovation, le régime sociotechnique et le paysage sociotechnique.

La niche représente le niveau microéconomique où les innovations radicales émergent et se développent (Geels et Schot, 2007). Ces innovations sont le plus souvent instables, portées par un nombre réduit d'acteurs, et ne disposent pas encore d'un cadre institutionnel favorable à leur expansion. Les niches sont considérées comme des chambres d'incubation qui protègent les innovations des forces de sélection du marché.

Le régime sociotechnique est l'environnement institutionnel composé de règles régulatrices (régulations, lois), normatives (valeurs, normes de comportements) et cognitives (systèmes de croyance). L'interprétation de ces règles (formelles et informelles) par les acteurs contribue soit à faciliter l'insertion et le déploiement de l'innovation dans le régime, soit à bloquer son expansion. La transition se produit lorsque l'on passe d'un régime sociotechnique à un autre (Geels et Schot, 2007).

Enfin, le paysage sociotechnique renvoie à l'environnement institutionnel qui influence le développement des niches à travers des pressions exercées sur le régime. Il se compose des facteurs externes au régime tels que: les tendances démographiques, les attentes sociétales en matière de production d'aliments, les politiques macroéconomiques (la demande croissante des agro-industries européennes pour le cacao durable), les considérations environnementales, les représentations culturelles. Ces facteurs vont agir d'une part sur la niche en incitant les acteurs à créer des innovations, et d'autre part sur le régime en faisant pression pour modifier et/ou compléter la dynamique déjà existante.

En fonction du niveau de développement de la niche et de l'intensité de la pression exercée par le paysage sociotechnique sur le régime, Geels et Schot (2007) proposent quatre chemins de transition possibles que sont (i) la transformation, (ii) la reconfiguration, (iii) la substitution technologique, et (iv) le désalignement et réalignement. La transformation se produit lorsque des pressions modérées du paysage sociotechnique poussent les acteurs du régime à faire des ajustements en faveur d'une niche peu développée. Si la niche est plus développée et qu'elle accomplit à peu près les mêmes fonctions que le régime, les acteurs du régime peuvent l'adopter comme complément pour résoudre des défaillances du régime. Il se produit un phénomène de reconfiguration. Dans le cas d'une niche développée, mais dont les fonctions diffèrent de celles du régime, les acteurs du régime peuvent décider de la soutenir (financièrement, politiquement, culturellement) pour la substituer au régime existant. Enfin, le phénomène de désalignement et réalignement se produit lorsque les pressions du paysage créent une perturbation forte au niveau du régime (désalignement). De nombreuses innovations émergent de la niche et entrent dans le régime. Au bout d'un certain temps, une finira par prendre le dessus et les autres s'aligneront derrière elle (réalignement).

Le rejet du cacao camerounais des ports européens montre que le paysage sociotechnique exerce une forte pression 
sur le régime ; ce qui exclut la possibilité d'un chemin de transformation résultant d'une pression modérée. Face à cette pression, des mesures semblables à la certification sont prises au niveau du régime pour produire durablement du cacao de qualité. Nous émettons l'hypothèse d'un chemin possible de reconfiguration, qui supposerait d'intégrer la certification à ces mesures pour une meilleure synergie d'actions.

\section{Méthodologie}

D'un point de vue méthodologique, l'économie évolutionniste (l'un des fondements dans lequel nous retrouvons les travaux sur les transitions sociotechniques) s'est très souvent appuyée sur des études de cas (Beckmann et al., 2009 ; Bennett et Elman, 2006 ; Gerring, 2004). Cette démarche repose sur l'analyse qualitative d'un petit nombre d'unités (pays, entreprises, groupes, individus, régions, partis, etc.). Les données sont collectées auprès de différentes sources (documents, entretiens ou enquêtes, observation participante), et le principal moyen de vérifier les données est la triangulation (Gerring, 2004).

Nous situons notre étude de cas dans la région Centre du Cameroun, l'un des principaux bassins de production du cacao (Minepat, 2014). L'étude de cas a débuté par la revue de deux documents: (i) le protocole de certification UTZ (UTZ, 2015) qui nous a permis de comparer le mode de fonctionnement théorique de la certification à son application pratique, (ii) un rapport de recherche (IITA, 2016) qui référence les initiatives nationales déjà existantes de promotion du cacao durable. Par la suite, onze entretiens semi-directifs et approfondis (une heure et demie en moyenne) ont été conduits auprès d'acteurs de la filière cacao. Les entretiens portaient sur les éléments présentés dans le tableau 1.

\section{Tableau 1. Informations obtenues au cours des entretiens}

\begin{tabular}{|c|c|c|}
\hline Sources d'informations & $\begin{array}{c}\text { Nombre } \\
\text { d'entretiens }\end{array}$ & Informations obtenues \\
\hline $\begin{array}{l}\text { Les responsables de deux coopératives de } \\
\text { producteurs de cacao certifié : MBANGASSUD } \\
\text { (Société Coopérative des producteurs de cacao } \\
\text { de Mbangassina Sud) et SOCAMAK (Société } \\
\text { Coopérative des producteurs de la Mefou et } \\
\text { Akono) }\end{array}$ & 4 & $\begin{array}{l}\text { - Le fonctionnement des coopératives } \\
\text { - Le déroulement du processus } \\
\text { de certification } \\
\text { - Les difficultés rencontrées } \\
\text { dans la certification. } \\
\text { - Le montant de la prime }\end{array}$ \\
\hline $\begin{array}{l}\text { Deux exportateurs: } \\
\text { AMS (Agroproduce Management Services) et } \\
\text { SIC CACAO (Société Industrielle Camerounaise } \\
\text { du Cacao) }\end{array}$ & 2 & \\
\hline $\begin{array}{l}\text { Le responsable en suivi-évaluation des projets de } \\
\text { certification UTZ (basé aux Pays-Bas) }\end{array}$ & 1 & $\begin{array}{l}\text { - Le niveau d'implication d'UTZ au } \\
\text { Cameroun } \\
\text { - Le rôle joué par UTZ dans la fixation } \\
\text { du montant de la prime }\end{array}$ \\
\hline $\begin{array}{l}\text { Un responsable de la coopération néerlandaise } \\
\text { (SNV), un du Ministère de l'Agriculture et du } \\
\text { Développement Rural (MINADER), et un du } \\
\text { Conseil Interprofessionnel du Cacao et du Café } \\
\text { (CICC) }\end{array}$ & 3 & $\begin{array}{l}\text { - Le rôle joué par les agences de } \\
\text { coopération et les services publics } \\
\text { dans la certification } \\
\text { - La perception qu'ont ces structures } \\
\text { de la certification }\end{array}$ \\
\hline $\begin{array}{l}\text { Un chercheur de I'International Institute of } \\
\text { Tropical Agriculture (IITA) }\end{array}$ & 1 & $\begin{array}{l}\text { - Les initiatives en cours de promotion } \\
\text { du cacao durable }\end{array}$ \\
\hline
\end{tabular}


L'étude de cas s'est achevée par l'administration d'un questionnaire à 90 producteurs, dont 44 certifiés et 46 non certifiés. Les producteurs choisis par effet boule de neige au sein des localités d'implantation des coopératives étaient interrogés sur leurs perceptions des obstacles au développement de la certification. Les coopératives choisies sont des acteurs incontournables de la production du cacao dans la région, de par leur nombre de membres et des superficies ou localités couvertes. Mbangassud compte 350 membres dont 280 sont certifiés Rainforest Alliance et UTZ confondus, et couvre six localités dans la région. Socamak est composée de 634 membres (dont 434 certifiés UTZ) et couvre près de 1215 hectares de plantation. Les deux coopératives rassemblent 6,5\% des 15000 producteurs de cacao répartis sur le territoire national (Bagal et al., 2013), et qui ont produit 253510 tonnes de cacao en $2018^{1}$. La durée limitée de cette étude (4 mois) n'a pas permis d'élargir notre échantillon d'enquête et constitue de ce fait une limite.

Les entretiens ont été entièrement retranscrits dans Microsoft Word, puis analysés dans Microsoft Excel, en classant les informations par thèmes (voir colonne « informations obtenues» du tableau 1). Les données issues des enquêtes agriculteurs ont elles aussi été saisies et analysées dans Microsoft Excel.

\section{Résultats et discussion}

\section{Les acteurs de la certification et leurs rôles}

\section{La certification : innovation radicale ou incrémentale?}

UTZ Certified est un programme et un label pour les producteurs et Acteurs de

1. Site officiel de l'Office national du cacao et du café (ONCC) : http://www.oncc.cm/index.php/ fr/2016-05-11-22-17-48/2016-05-30-13-58-58/ statistiques-cacao. la chaîne d'approvisionnement (ACA) qui souhaitent vendre et/ou acheter leur produit comme étant certifié UTZ (UTZ, 2015). L'objectif d'UTZ est d'améliorer la qualité sociale de la production de cacao en conformité avec les normes internationales de l'Organisation internationale $\mathrm{du}$ travail. UTZ incite les producteurs à l'usage de bonnes pratiques agricoles telles que l'interdiction d'utiliser des pesticides prohibés, la production du cacao dans un système agroforestier comportant au moins 18 arbres/ha, la réduction du taux d'humidité des fèves à $7 \%$. Outre les pratiques agricoles, UTZ met l'accent sur des normes sociales telles que l'obligation pour les employés de l'exploitation de disposer d'un contrat de travail, l'interdiction pour les mineurs (moins de 18 ans) d'effectuer des tâches dangereuses ou susceptibles de compromettre leur santé physique ou leur bien-être moral.

Les entretiens avec des exportateurs et les responsables du système UTZ ont révélé qu'avant l'arrivée de la certification au Cameroun, les exportateurs achetaient déjà le cacao directement aux coopératives. Ils contribuaient à mettre à la disposition des producteurs (par l'intermédiaire de leurs coopératives) des crédits de campagne (sans intérêts) pour la production du cacao. Les producteurs en retour avaient l'obligation de livrer toute leur production à la coopérative qui l'acheminait vers l'exportateur. Dès le début des années 2000, conscients des nouvelles exigences du marché international, les exportateurs décident après accord avec les producteurs d'introduire la certification comme gage de production d'un cacao durable et de qualité. C'est ainsi que les activités de certification débutent au Cameroun en 2010. Les exportateurs ont d'abord introduit le système Rainforest Alliance, puis ont décidé d'y ajouter la norme UTZ pour rassurer davantage les acheteurs et cibler un plus grand marché. Par la suite, les systèmes Fairtrade et Organic ont aussi fait 
leurs entrées, mais UTZ reste le système dominant au moment de l'étude. En 2012, le Cameroun a produit 2799 tonnes de cacao certifié UTZ, soit $0,5 \%$ de la production mondiale UTZ (535000 tonnes) (Potts et al., 2014).

La certification UTZ est mise en œuvre par des acteurs dominants déjà présents dans le régime sociotechnique. Ces derniers décident d'un changement pour s'adapter aux nouvelles normes de compétitivité que fixe la régulation du marché international des produits de base (Daviron et Gibbon, 2002). La certification n'est pas, dans notre cas, une innovation de niche portée par des acteurs isolés en quête de stabilité et/ou de reconnaissance. Ce résultat correspond à celui de Elzen et al. (2012) qui montre que certaines innovations se développent dans un espace à mi-chemin entre la niche et le régime. Cela est dû au fait que, d'une part, ces innovations rassemblent des acteurs qui appartiennent à la fois à la niche et au régime (exportateurs et coopératives en l'occurrence) et, d'autre part, l'innovation crée un ancrage institutionnel avec les pratiques déjà existantes dans le régime. Nous verrons dans les sections suivantes que les pratiques liées à la certification ne constituent pas en elles-mêmes une rupture par rapport à la dynamique émergente du régime. Des initiatives de promotion des bonnes pratiques agricoles existent et sont promues par les organismes de recherche. La certification ne constitue qu'un mécanisme complémentaire d'un changement plus profond. Elle pourrait, par le mécanisme de la prime, inciter davantage les producteurs à s'orienter vers une production durable.

Fonctionnement général de la certification Dans la certification UTZ, les producteurs certifiés (représentés par leur coopérative) travaillent principalement avec les exportateurs (organismes de certification) dans la mise en pratique des exigences du label. Une différence est faite dans le protocole de certification d'UTZ entre les Organismes de certification (OC) et le programme de certification UTZ. Les Organismes de certification sont des structures ou des personnes chargées de mettre la certification en pratique sur le terrain. $\mathrm{Ce}$ sont essentiellement les exportateurs et les auditeurs. UTZ n'interagit pas directement avec les producteurs. Les exportateurs se chargent de contacter les auditeurs agrées UTZ qui après l'audit envoie un rapport de la situation de la coopérative à UTZ. Ce dernier élabore le certificat qui est remis à la coopérative par l'exportateur. Ce mode de fonctionnement est conforme à la procédure de certification décrite dans le protocole d'UTZ, et repris dans l'encadré 1 .

La certification se caractérise par une interaction entre quatre principaux acteurs : les producteurs ou groupes de producteurs, les exportateurs et auditeurs agréés UTZ, et UTZ (figure 1).

Les agences de coopération néerlandaise (Stichting Nederlandse Vrijwilligers (SNV)) et allemande (Gesellschaft für Internationale Zusammenarbeit (GIZ)), les centres de recherche et les services publics ne sont pas directement impliqués dans la certification. Ces structures travaillent avec les producteurs pour diverses raisons (formation, mise à disposition des plants de cacao), sans qu'un système de certification n'y soit adossé. Les coxeurs sont, quant à eux, des acheteurs non agréés de cacao, qui préfinancent la production des cacaoculteurs, en échange d'une quantité proportionnelle de cacao. Une description détaillée des relations entre chaque groupe d'acteurs est faite dans les sections suivantes.

Gestion mutuelle de la qualité : relation producteurs-coopérative

Les coopératives agricoles sont des acteurs clés du processus de certification; elles sont chargées de faire respecter les normes $\mathrm{du}$ système UTZ par leurs membres. Être membre d'une OP est d'ailleurs 


\section{Encadré 1. Procédure générale de certification UTZ}

- Un nouveau membre s'inscrit en remplissant le formulaire d'inscription sur le site web UTZ Certified.

- UTZ fournit au nouveau membre la confirmation de l'inscription dans le GIP (Good Inside Portal).

- Le membre choisit et contacte un OC (exportateurs ou auditeurs). Ils signent un contrat.

- L'OC fournit la dernière version de tous les documents pour la certification UTZ (Protocole de Certification) afin que le membre les lise et les comprenne.

- Le membre et l'OC conviennent de la date de l'audit.

- L'OC réalise l'audit annuel selon les exigences du Code de conduite UTZ.

- Si des non-conformités sont trouvées lors de l'audit, le membre met en œuvre une mesure corrective pour les résoudre.

- L'OC réalise un autre audit pour confirmer que les non-conformités ont été résolues.
- Si l'OC décide de ne pas certifier le membre, l'OC informe le membre et UTZ Certified de la décision.

- Si l'OC décide de certifier le membre, l'OC demande une licence pour le membre dans le GIP dans un délai de 5 jours ouvrés après la décision de certification.

- UTZ vérifie les informations envoyées par l'OC dans un délai de 5 jours ouvrables.

- Si UTZ estime que les informations dans la demande de licence sont incorrectes, incomplètes ou ne sont pas claires, une clarification par l'OC dans le rapport d'audit et/ou la demande de licence sont demandées.

- L'OC clarifie, corrige et/ou complète les informations dans la demande de licence, et la renvoie à UTZ dans un délai de 7 jours ouvrable.

- Si UTZ considère que les informations dans la demande de licence sont correctes et complètes, il active la licence dans le GIP.

- L'OC envoie le certificat au membre; le certificat est valable un an.

Source : UTZ (2015). une condition nécessaire pour adhérer à un programme de certification. Tous les Producteurs certifiés (PC) qui ont fait l'objet de notre enquête sont membres d'une OP, comparativement aux Producteurs non certifiés (PNC) dont la moitié seulement en fait partie (tableau 2).

Les organismes de certification ciblent les OP fonctionnant bien (Hütz-Adams et al., 2016 ; Ingram et al., 2014 ; Ruf et al., 2013), c'est-à-dire en mesure de rendre divers services à leurs membres (achat d'intrants, collecte et commercialisation des produits, etc.) sans compromettre la solvabilité et la viabilité de l'organisation. Ntsama et Kamgnia (2008) ont mis en lumière le rôle positif des OP dans l'adoption d'innovations, à condition que ces OP aient une représentativité importante et qu'elles constituent une structure reconnue comme interlocuteur valable auprès des partenaires. S'agissant de leur fonctionnement, $79 \%$ des producteurs certifiés que nous avons interrogés estiment que l'OP tient des réunions régulièrement, $72 \%$ pensent qu'elle les tient informés de toutes les activités, et $63 \%$ qu'elle les aide véritablement à vendre leur cacao au meilleur prix.

La coopérative Mbangassud est un exemple intéressant d'action collective et d'intégration verticale dans la filière cacao certifié. Mbangassud débute les activités de certification en 2012, sous recommandation de son acheteur, Sic Cacao, avec qui elle entretenait déjà des relations. Elle obtient le certificat Rainforest Alliance en 2012 et celui d'UTZ en 2016. La coopérative permet aux producteurs certifiés d'externaliser de nombreux coûts. Elle assure leur formation à travers des producteurs relais qui ont été formés par Sic Cacao (acheteur et exportateur certifié UTZ). Mbangassud dispose de six producteurs 
Figure 1. Organisation de la certification UTZ du cacao dans la région du Centre

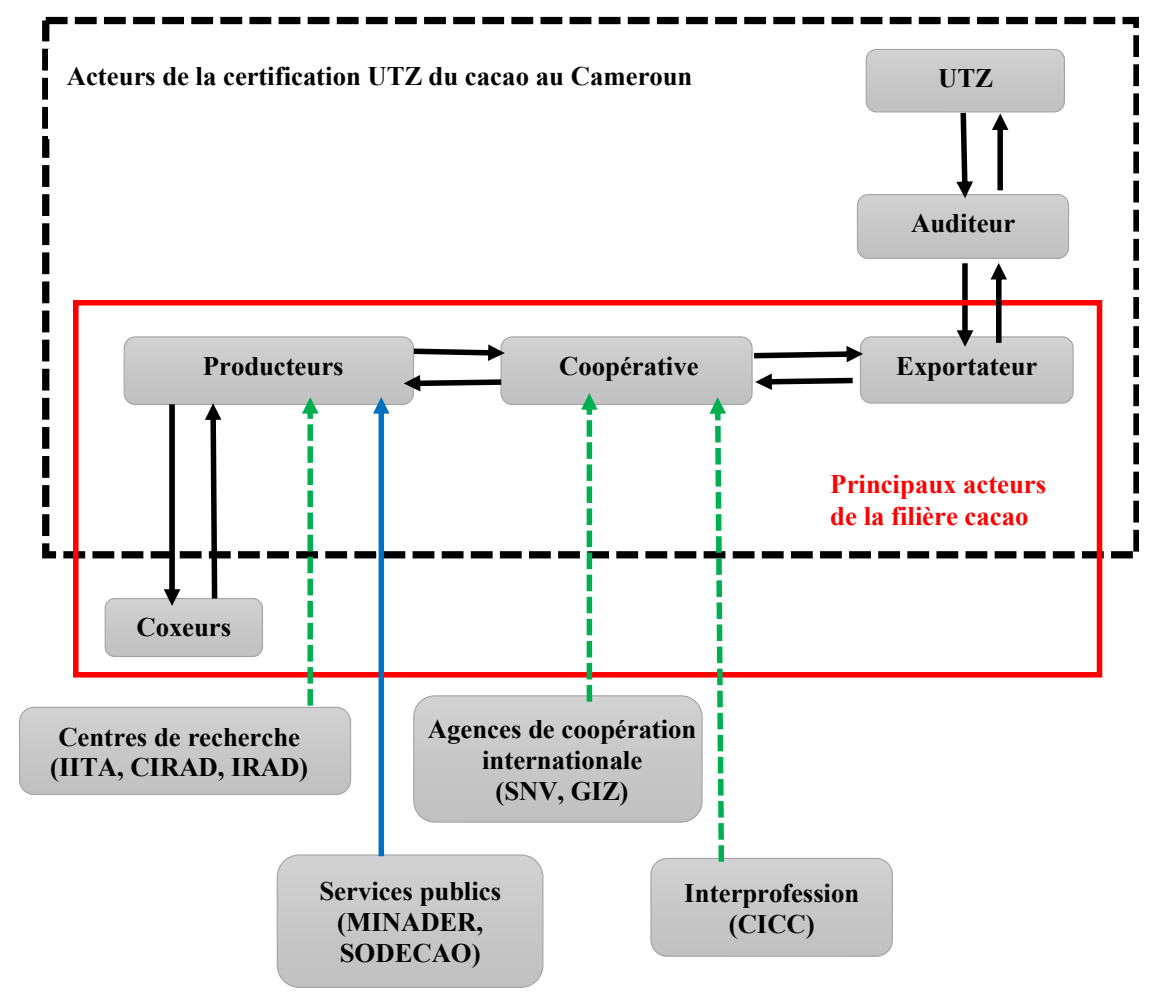

Relation en lien avec la certification

Formation des producteurs aux bonnes pratiques agricoles sans lien avec la certification

Mise à disposition des plants de bonne qualité aux producteurs

Source : les auteurs.

Tableau 2. Caractérisation des producteurs enquêtés selon leur appartenance à une OP

\begin{tabular}{lccc}
\hline & PNC & PC & Total \\
\hline Non-membre d'une OP & $22(47,83 \%)$ & 0 & 22 \\
Membre d'OP & $24(52,17 \%)$ & $44(100 \%)$ & 68 \\
Total & 46 & 44 & 90 \\
\hline
\end{tabular}

Source : les auteurs.

relais répartis dans les six villages dans lesquels la coopérative est active. Les producteurs relais sont des membres de la coopérative chargés de contrôler la mise en pratique des bonnes pratiques agricoles des producteurs certifiés et éventuellement sensibiliser les producteurs non certifiés à adhérer à la certification. Une fois les producteurs certifiés formés, la coopérative prend contact avec l'acheteur pour demander un audit des exploitations de la coopérative. Le président du Conseil d'administration adresse un mail à Sic Cacao qui contacte à son tour la représentation 


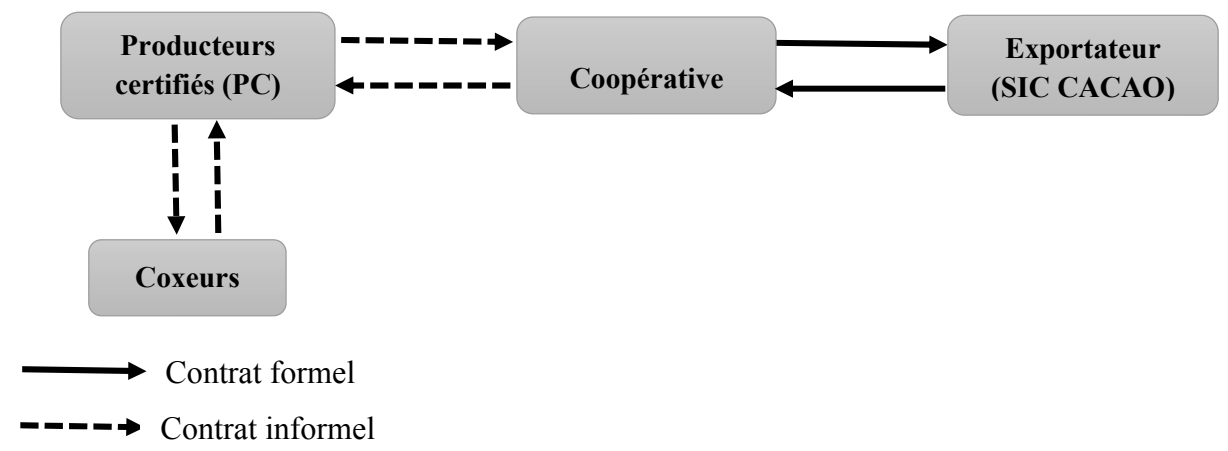

Source : les auteurs.

UTZ basée en Afrique de l'Ouest (Côte d'Ivoire). Les coûts d'audit et de formation sont prélevés de la prime des producteurs d'un montant de $30 \mathrm{FCFA} /$ producteur/kg de cacao. Après déduction de ces coûts, la prime versée aux producteurs certifiés est de $40 \mathrm{FCFA} /$ producteur/kg de cacao. Du fait de la certification, la coopérative a en son sein des «brigadiers phyto », dont le rôle est d'aider les producteurs certifiés qui les sollicitent dans le traitement de leurs cultures et la fourniture de services de conseil dans ce domaine. Leur travail est rémunéré à $50 \%$ par la coopérative et à $50 \%$ par le producteur qui sollicite leurs services.

La coopérative achète de façon groupée les produits phytosanitaires des producteurs certifiés et se charge de leur redistribution. Elle collecte le cacao de ses membres, le stocke et l'achemine vers l'entrepôt de l'exportateur. La négociation des termes du contrat avec l'acheteur/ exportateur fait aussi partie intégrante des services rendus par la coopérative à ses membres. En effet, le rassemblement des producteurs sous forme de coopérative leur permet de négocier collectivement leur contrat (Bouamra-Mechemache et al., 2014) et de réduire l'asymétrie dans les négociations entre le maillon agricole et le maillon de transformation (Zeuli et King, 2004). Cela favorise la réduction de l'incertitude, puisque le producteur sait à l'avance à qui il vendra son cacao.

La relation entre les producteurs et leur coopérative est caractérisée par un contrat verbal (N'Dao, 2012) dans lequel le producteur doit livrer entièrement sa production à la coopérative pour bénéficier des services de la coopérative. Mais, ce contrat n'est pas toujours respecté car le producteur livre une partie de sa production aux coxeurs. En effet, pour financer la production ou résoudre des soucis pressants (rentrée scolaire imminente, cas de maladie, décès d'un proche, etc.), il arrive que le producteur emprunte de l'argent aux coxeurs qui sera remboursé en quantité équivalente de kilogrammes de cacao produits. La relation qui lie le producteur certifié à sa coopérative est matérialisée par la figure 2 .

\section{Relation contractualisée : relation coopérative-exportateur}

Les exportateurs constituent aussi un maillon central du processus de certification. Ce sont eux qui, du fait de la pression subie par les acheteurs étrangers, incitent les producteurs à adhérer à la certification. Il s'agit de relations d'agence dans lesquelles 
le principal est l'exportateur et les coopératives sont les agents. Les exportateurs sont des acheteurs certifiés UTZ qui ont des départements dédiés à la certification et aux champs écoles paysans, et qui établissent des relations commerciales avec les coopératives chargées de produire du cacao conformément aux normes de certification (Ouattara, 2015). Les contrats signés avec les coopératives sont à la fois des contrats de production et de commercialisation, puisqu'ils portent à la fois sur les procédés de production, mais aussi sur le prix, les volumes, les dates de livraison et la destination (Vavra, 2009). Dans cette étude, nous avons rencontré deux exportateurs, AMS et Sic Cacao, dont le mode de collaboration avec les coopératives est différent.

\section{Relation Sic Cacao et Mbangassud}

Sic Cacao est une filiale de Barry Callebault, qui achète à la fois les fèves de cacao certifié et non certifié, les transforme en poudre de cacao et en produits semi-finis. Il vend une partie des fèves à Chococam (Chocolaterie et Confiserie du Cameroun) et l'autre partie - la plus importante - à Barry Callebault. La relation entre Sic Cacao et Mbangassud est matérialisée par des contrats formels d'exclusivité d'une durée de trois ans renouvelable. La coopérative n'a pas de relation directe avec l'organisme de certification UTZ, celui-ci n'ayant pas de représentation au Cameroun. Toutes les activités liées à la certification passent par l'exportateur qui relaie l'information au bureau UTZ de Côte d'Ivoire. Tout producteur désirant adhérer à la certification adresse une demande à Sic Cacao qui impose alors à la coopérative d'organiser des champs écoles paysans, dès lors que le seuil minimum de producteurs est atteint. Les producteurs relais forment les nouveaux adhérents et vérifient, à travers des inspections, qu'ils mettent bien en pratique les enseignements reçus. Après l'audit, le certificat est délivré au nom de la coopérative (certificat de groupe).

Sic Cacao intervient aussi bien en aval, qu'en amont de l'activité cacaoyère. En amont, il préfinance $80 \%$ des coûts de certification (les $20 \%$ restants étant à la charge de la coopérative). Ces charges se composent du paiement mensuel des six producteurs relais (100 000 FCFA/mois/ personne) et des frais d'audits annuels. En aval, elle distribue aux producteurs certifiés leur prime conformément à l'arrangement signé avec la coopérative et après déduction des coûts de certification. Ce mode d'organisation des transactions est en adéquation avec l'idée selon laquelle la coordination verticale dans les filières peut permettre au producteur de se prémunir de différents risques (Bouamra-Mechemache et al., 2014 ; Tankam, 2017).

Il existe deux types de contrats signés entre la coopérative et Sic Cacao: un contrat d'achat du cacao ordinaire et un contrat lié à la certification. Le tonnage du premier contrat est déterminé par la coopérative et peut fluctuer selon les années, en fonction des capacités de production des membres (aussi liées aux aléas climatiques). En revanche, pour le second contrat, la coopérative ne peut influencer le tonnage demandé, celui-ci étant déterminé par les acheteurs étrangers. En début de campagne, Sic Cacao informe la coopérative de la quantité de cacao certifié demandée par les acheteurs, et il arrive quelquefois que l'excédent de cacao certifié produit par la coopérative ne puisse être acheté en tant que cacao certifié. Si cette quantité n' augmente pas et que les producteurs certifiés actuels sont en mesure de satisfaire cette demande, la coopérative ne peut transmettre à Sic Cacao les demandes de certification de nouveaux producteurs, sachant bien que leur cacao certifié sera acheté en cacao ordinaire et qu'ils ne recevront pas la prime. Près de $5 \%$ des producteurs non certifiés enquêtés ont d'ailleurs 
admis avoir démarré le processus de certification depuis des mois, certains des années, mais leurs noms ne sortent jamais sur les listes des producteurs certifiés. La coopérative dispose d'un faible pouvoir de négociation (Mithöfer et al., 2017) dans les contrats de certification. Le fait que les acheteurs ne garantissent pas l'achat avec prime de l'intégralité du cacao certifié constitue un obstacle à la participation des producteurs aux programmes de certification.

\section{Relation AMS et Socamak}

Agroproduce Management Services (AMS) est une filiale basée aux Pays-Bas de l'entreprise Theobroma, leader indépendant dans l'achat et la vente de cacao. C'est la première organisation qui a débuté les activités de certification avec UTZ au Cameroun. Socamak est certifiée UTZ depuis 2012, sous l'impulsion de Facoko (un autre acheteur) qui au moment de l'enquête ne travaillait plus au Cameroun. Facoko quitte le Cameroun en 2014, en laissant la coopérative sans un acheteur à qui livrer la production. À l'arrivée d'AMS en milieu d'année 2015, la coopérative est déstabilisée et fragilisée à cause du nombre important de membres qui préfèrent traiter avec les coxeurs, plutôt qu'avec la coopérative. Le partenariat avec AMS apparaît comme un souffle nouveau pour la coopérative et un moyen de regagner la confiance de ses membres.

Cependant, l'analyse de la relation entre AMS et Socamak laisse apparaître une perte d'autonomie de la coopérative. À Socamak, les producteurs certifiés sont formés par AMS et non par des producteurs relais. C'est aussi AMS qui s'occupe de la collecte des fèves de cacao auprès des producteurs, et de leur acheminement vers le magasin de stockage de la coopérative. La coopérative en elle-même n'existe presque plus, puisque AMS, acheteur exclusif, internalise tous les coûts liés à la certification. Ces coûts (audits et formation) sont par la suite déduits du montant de la prime au prix de $30 \mathrm{FCFA} / \mathrm{kg} /$ producteur ; le montant restant de la prime étant de 40 FCFA $/ \mathrm{kg} /$ producteur. L'acheteur courtcircuite la coopérative pour signer des contrats directement avec les producteurs.

La coopérative ne possède pas de certificat UTZ; ce dernier est délivré au nom d'AMS qui présente ses producteurs à la certification. Cette façon de faire lie la coopérative à l'acheteur et génère une forte dépendance des coopératives certifiées visà-vis des acheteurs. En cas de rupture de contrat avec AMS, les producteurs, et la coopérative elle-même, ne peuvent pas justifier d'un document qui atteste des bonnes pratiques appliquées et de la qualité du cacao produit auprès d'un autre acheteur. Ce résultat correspond à celui trouvé par N'Dao (2012) qui affirmait qu'en pratique, la coopérative, malgré sa position d'intermédiaire entre les producteurs et l'exportateur, n'est pas en réalité le principal acteur dans le mécanisme contractuel : «Certes, le contrat est signé entre le producteur et la coopérative mais dans les faits, c'est entre le producteur et l'exportateur » (N'Dao, 2012). La relation entre AMS et Socamak est matérialisée par la figure 3.

Bien que Mbangassud soit plus structurée et fonctionnelle que Socamak, les deux coopératives disposent d'un faible pouvoir de négociation avec les acheteurs, en partie due au fait que le cacao soit une denrée peu ou pas consommée par les producteurs, et donc forcément vendue dans son intégralité. L'entretien avec le responsable UTZ des projets de certification a révélé que le montant de la prime doit être déterminé par arrangement entre les producteurs et l'acheteur; UTZ n'y exerce aucune influence. Or, du fait de l'exclusivité des contrats, les producteurs représentés par leur coopérative sont des preneurs de prix, ils subissent le prix imposé par l'acheteur. En outre, l'ingérence de ce dernier dans la vie de la coopérative limite la mise en relation avec d'autres acheteurs, et génère de 


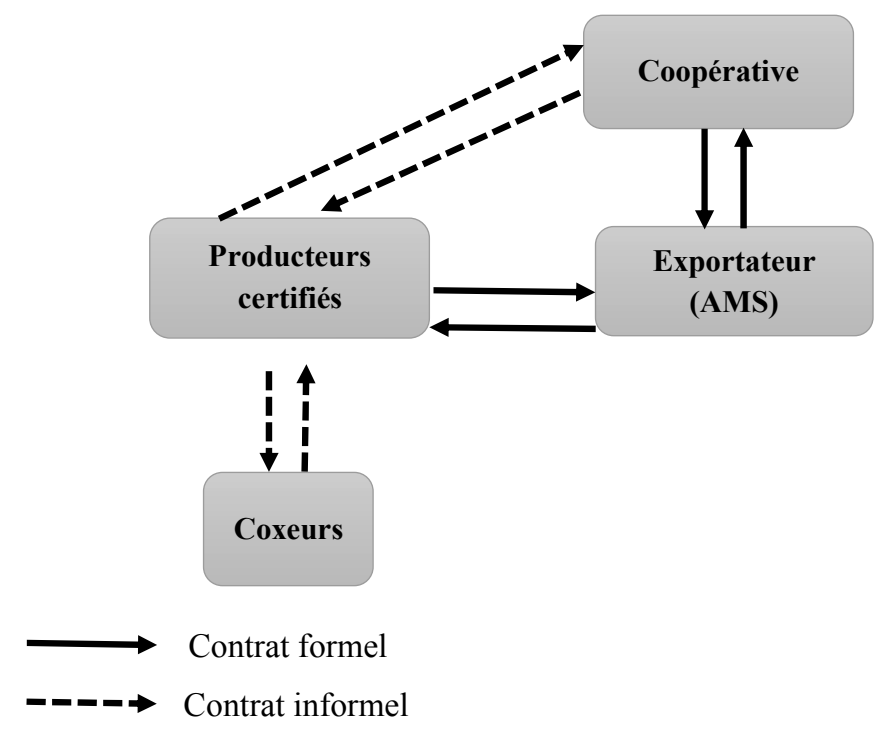

Source : les auteurs.

la dépendance. Les stratégies concurrentielles des acheteurs constituent donc un obstacle au déploiement de la certification. En effet, le faible montant de la prime et l'absence d'achat en intégralité du cacao certifié produit entraînent une faible participation des producteurs aux programmes de certification.

\section{Les entraves à la certification}

\section{Absence de partenariats public-privé} dans la certification

L'entretien avec un responsable du ministère de l'Agriculture (Minader) a révélé que la certification au Cameroun est une entreprise privée dans laquelle le secteur public n'interfère pas. Or les entreprises privées font aussi face à des charges de fonctionnement importantes et ne peuvent couvrir toutes les zones au même moment. En outre, les enquêtes auprès des producteurs ont révélé que la principale raison de nonadhésion des producteurs aux programmes de certification est le manque d'information.
Certains en ont vaguement entendu parler à la radio, mais ils ne maîtrisent pas ses réels avantages et la procédure à suivre pour se faire certifier. L'un des obstacles au développement de la certification est donc l'absence de partenariats public-privé dans ce secteur. Cela confirme l'hypothèse de l'étude selon laquelle la certification UTZ au Cameroun est une innovation incrémentale qui repose sur un nombre restreint d'acteurs du secteur privé. Elle modifie peu les institutions et politiques publiques qui régulent le secteur cacao.

Pourtant, la proximité géographique des agents du Minader déployés en zones rurales contribuerait à informer les producteurs des enjeux liés à la certification, puisque l'accompagnement des producteurs fait partie de leurs fonctions régaliennes. Il a déjà été démontré que le contact des producteurs avec les services publics de vulgarisation augmente de manière significative la probabilité d'adopter une innovation (Mabah Tene et al., 2013) 
et que l'accès à l'information sur la certification était nécessaire pour percevoir ses effets positifs (Fenger et al., 2017). Les partenariats public-privé pourraient aussi contribuer à orienter les programmes gouvernementaux vers des trajectoires de durabilité des chaînes de valeur (Ingram et al., 2018). Dans une approche systémique de l'innovation, le sous-système d'intermédiation représenté par le ministère de l'Agriculture et les structures parapubliques (CICC, SODECAO) a aussi son rôle à jouer dans la mise en relations des acteurs (Temple et al., 2017).

\section{Faible capacité des $O P$ à négocier des contrats équitables}

Du fait de leur rôle clé dans le processus de certification, les OP pourraient avoir de l'influence dans les négociations avec les entreprises, et peuvent grâce à leurs immobilisations (magasin de stockage) servir de garantie pour l'accès des producteurs au crédit. Pourtant, la réalité est tout autre : les institutions financières hésitent à prêter au monde agricole, en raison du risque élevé réel et/ou perçu (Huybrechts, 2007). Pour accéder à des financements, les cacaoculteurs n'ont pas d'autre possibilité que de vendre aux coxeurs qui préfinancent la production (Fenger et al., 2017 ; Van Rijsbergen et al., 2016), ou dans le cas des coopératives de signer des contrats exclusifs avec des exportateurs. Les exemples de Socamak et de Mbangassud avec l'organisation oligarchique de l'aval sont une raison du faible déploiement de la certification dans le régime sociotechnique. Il faudrait améliorer le pouvoir de négociation des coopératives, en les rendant indépendantes vis-à-vis des acheteurs (Huybrechts, 2007) et en multipliant les acheteurs. Mais dans le cadre d'un produit agricole plus destiné à satisfaire le marché extérieur qu'intérieur, la marge de manœuvre des coopératives reste limitée et on pourrait vite arriver à un blocage dans les négociations.

\section{Pas de différence de prix entre \\ le cacao certifié et le cacao ordinaire}

Les producteurs non certifiés affirment que, sur le marché, il n'existe pas de différence réelle de prix entre le cacao ordinaire et le cacao certifié. Selon eux, le seul avantage perçu par les producteurs certifiés est la prime, qu'ils ne jugent pas suffisamment incitative en comparaison des efforts fournis par le producteur. En outre, l'évolution décroissante (de 50 à 40 FCFA entre 2015 et 2016) du montant de la prime pousse à s'interroger sur l'intérêt des producteurs à la certification à la disparition de la prime. Les entretiens ont révélé que l'objectif de la certification est qu'à terme il n'y ait plus de prime car les producteurs auront déjà intégré les bonnes pratiques agricoles qui leur permettront d'accroître leurs rendements et de préserver l'environnement. L'importance accordée par les producteurs aux enjeux de productivité et de rentabilité, plutôt qu'à ceux de durabilité (Lemeilleur et al., 2015), font du prix du cacao certifié un critère déterminant au développement de la certification.

En l'absence d'une différenciation au niveau du prix, la certification n'apparaîtra pas pour les producteurs comme la solution à l'amélioration de leurs revenus et/ou de leurs conditions de vie (Defries et al., 2017), et ce d'autant plus que le marché du cacao n'est plus seulement l'apanage des acheteurs européens. Des entreprises japonaises et nigérianes se sont lancées depuis quelques années dans l'achat et la transformation du cacao. Elles achètent tous types de cacao sans distinction. La pression pour le cacao durable n'est donc pas encore suffisamment forte pour que les producteurs renoncent radicalement aux techniques de production conventionnelles.

\section{Quel avenir pour la certification ?}

\section{Des fenêtres d'opportunités pour la certification}

Depuis 2016, la coopération allemande (GIZ) travaille avec l'IITA, la SNV, L'Irad 
et le Minader dans la mise en place d'un projet de formation des producteurs de cacao aux bonnes pratiques agricoles. Le CICC, fédération de tous les acteurs privés des filières cacao et café, coordonne aussi un projet dont l'objectif général est d'engager les producteurs camerounais dans un processus de certification. Ces projets visent à améliorer la qualité du cacao, sans qu'un système de certification spécifique n'y soit adossé. Leurs curricula de formation sont rédigés en collaboration avec les organismes de certification (UTZ et Rainforest Alliance).

Bien qu'ils ne reçoivent aucune prime, les producteurs accompagnés par ces projets fournissent les mêmes efforts que les producteurs certifiés, en matière de production de cacao durable et de qualité. Si la littérature fait état de $3 \%$ de cacao certifié au Cameroun (Ngoucheme et al., 2016), il existe toutefois un pourcentage non encore répertorié de cacao de qualité. Ces projets constituent un ancrage, au sens d'Elzen et al. (2012) pour le développement de la certification, et sur lesquels les organismes de certification pourraient s'appuyer.

\section{Vers une possible reconfiguration du régime sociotechnique?}

Dans le contexte de l'innovation posée par la certification du cacao au Cameroun, notre analyse identifie une situation possible de reconfiguration. En effet, les changements de mode de consommation dans les pays européens et l'accentuation des mesures de préservation de l'environnement ont conduit au développement de la certification. Cette dernière est le fruit d'acteurs (exportateurs et producteurs) à mi-chemin entre la niche et le régime. Lorsque Mars et Barry Callebaut annoncent leur volonté de n'acheter que du cacao certifié en 2020, cela crée une perturbation supplémentaire dans le régime au moment où la certification est déjà développée. De par sa similitude avec les bonnes pratiques agricoles déjà promues dans le régime, la certification pourrait être adoptée comme complément à celles-ci. Les chercheurs (la science), les politiciens (la politique) et les producteurs dont les pratiques conventionnelles de production de cacao sont très encastrées (la culture) vont agir de concert pour faciliter son insertion et son déploiement. Le régime s'acheminerait avec le temps vers une reconfiguration, représentée par la figure 4. Mais, pour que cela soit possible, les acteurs de la certification devraient impliquer les services publics pour une plus large diffusion de l'innovation. De plus, des mesures ou politiques de prix en faveur d'un cacao de bonne qualité devraient être mises sur pied pour encourager les producteurs.

La figure 4 présente les perturbations fortes du paysage qui s'exercent à deux niveaux: (i) au niveau du régime, elles sont à l'origine de la mise sur pied de programmes et projets de production du cacao durable, (ii) à mi-chemin entre le régime et la niche, elles introduisent la certification comme gage de production d'un cacao de qualité. Ces pressions incitent les producteurs à se détourner de la cacaoculture conventionnelle, solidement implantée dans le régime, et à s'orienter vers un mode de production plus demandé sur les marchés européens. La certification qui porte un positionnement marqué pour la préservation de l'environnement pourrait être ajoutée comme complément aux initiatives déjà en cours pour rassurer davantage les acheteurs et redorer le blason du cacao camerounais. Nous aboutirions avec le temps à une reconfiguration du régime sociotechnique, qui est facilitée par la science, la culture et la politique. Les scientifiques doivent prouver aux producteurs le bien-fondé de la certification sur l'augmentation des rendements et des revenus. La culture en tant que véhicule des représentations sociales par le biais des conversations journalières, des médias, des coutumes, doit après acceptation des résultats de recherche répandre 


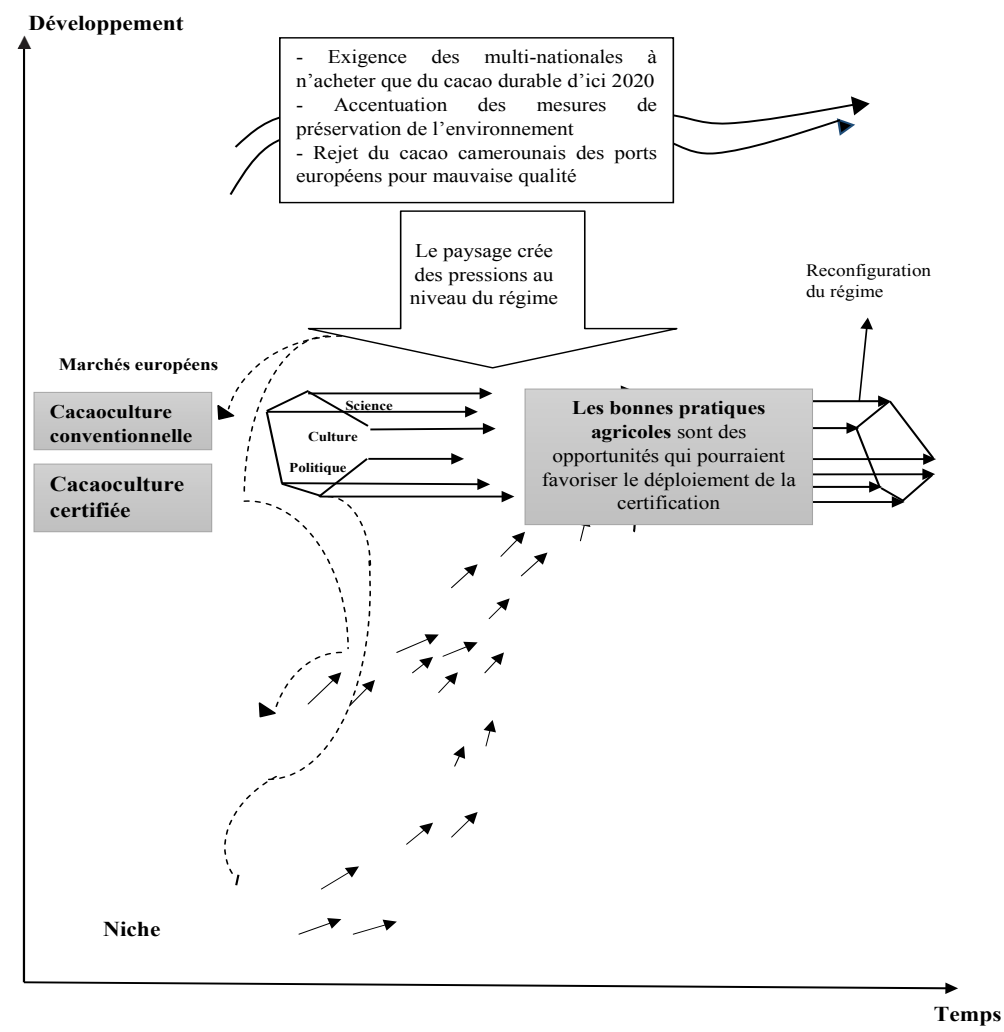

Source : adapté de Geels et Schot (2007).

ce nouveau mode de production. Enfin, les politiciens doivent définir des règles qui protègent les producteurs contre les forces de sélection du marché. Cela permettrait de répondre aux trois objectifs des innovations agricoles : accroître les rendements et les revenus des agriculteurs, préserver l'environnement et être acceptées par les agriculteurs eux-mêmes et par la société (Bellon-Maurel et Huyghe, 2016).

$$
\text { * } *
$$

L'objectif de cet article était d'identifier les déterminants d'adoption de la certification UTZ du cacao au Cameroun dans un contexte d'évolution des normes internationales. Ces déterminants comprennent entre autres : la mise en place de partenariats public-privé pour faciliter la dissémination de l'innovation, l'appartenance des producteurs à des OP bien organisées, une meilleure valorisation du travail supplémentaire lié à la mise en pratique des bonnes pratiques agricoles. Nous confirmons l'hypothèse de départ selon laquelle le processus actuel de certification étant de nature incrémentale et principalement porté par quelques acteurs privés, son efficacité dans la construction d'un changement de trajectoire technologique reste faible. La pertinence des normes de certification 
et de leurs conditions institutionnelles de mise en œuvre au Cameroun s'en retrouve interrogée. Néanmoins, les bonnes pratiques agricoles mises en pratique par certains producteurs (sans lien avec la certification) et la demande croissante des multinationales pour du cacao durable constituent des opportunités de développement de la certification.

En partant de l'exemple du Cameroun, l'étude montre à l'aide du modèle multiniveaux, l'intérêt d'une approche systémique pour comprendre le développement d'une innovation. L'analyse systémique des interactions entre le régime sociotechnique et le cadre macroéconomique est beaucoup plus structurante pour la compréhension du processus d'innovation que l'analyse classique des conditions d'implémentation des niches ou des mécanismes individuels d'adoption. Outre son rôle dans l'explication des déterminants à différents niveaux d'analyse, ce modèle permet aussi d'entrevoir la trajectoire possible d'une innovation sous réserve que certaines conditions soient réunies.

Cette étude a été financée par le ministère fédéral de la Coopération économique en Allemagne, dans le cadre du projet Potential Analysis of Innovative Approaches for the Cocoa value chain in Cameroon (PotinnocC). 


\section{RÉFÉRENCES BIBLIOGRAPHIQUES}

Bagal M., Belletti G., Marescotti A. (2013). Étude sur le potentiel de commercialisation du cacao du Cameroun en «Indication géographique». Rapport d'étude, Lausanne, REDD, Iram, p. 26.

Beckmann V., Padmanabhan M. (2009). Analyzing institutions: What Method to Apply? In Beckmann V., Padmanabhan M. (eds.), Institutions and Sustainability, Dordrecht, Springer, pp. 341-371.

Bellon-Maurel V., Huyghe C. (2016). L'innovation technologique dans l'agriculture. Géoéconomie, vol. 80, $\mathrm{n}^{\circ} 3$, pp. 159-180.

Bennett A., Elman C. (2006). Complex Causal Relations and Case Study Methods: The Example of Path Dependence. Political Analysis, vol. 14, $\mathrm{n}^{\circ} 3$, pp. 250-267.

Bouamra-Mechemache Z., DuvaleixTreguer S., Ridier A. (2014). Contrats et modes de coordination en agriculture. Working paper SMART-LERECO, vol. 7 , $n^{\circ} 14$.

Bui S., Cardona A., Lamine C., Cerf M. (2016). Sustainability transitions: Insights on processes of niche-regime interaction and regime reconfiguration in agri-food systems. Journal of Rural Studies, $\mathrm{n}^{\circ}$ 48, pp. 92-103.

Crossan M. M., Apaydin M. (2010). A MultiDimensional Framework of Organizational Innovation: A Systematic Review of the Literature. Journal of Management Studies, vol. 47, n 6, pp. 1154-1191.

Daviron B., Gibbon P. (2002). Global commodity chains and African export agriculture. Journal of Agrarian Change, vol. 2, $\mathrm{n}^{\circ} 2$, pp. 137-161.

DeFries R. S., Fanzo J., Mondal P., Remans R., Wood S. A. (2017). Is voluntary certification of tropical agricultural commodities achieving sustainability goals for smallscale producers? A review of the evidence. Environmental Research Letters, vol. 12, $\mathrm{n}^{\circ} 3,033001$.

Elzen B., Van Mierlo B., Leeuwis C. (2012). Anchoring of innovations: assessing Dutch efforts to harvest energy from glasshouses.
Environmental Innovation and Societal Transitions, $\mathrm{n}^{\circ}$ 5, pp. 1-18.

Fenger A. N., Bosselmann S. A., Asare R., De Neergaard A. (2017). The impact of certification on the natural and financial capitals of Ghanaian cocoa farmers. Agroecology and Sustainable Food Systems, vol. 41, n ${ }^{\circ} 2$, pp. 143-166.

Geels F. W., Schot J. (2007). Typology of sociotechnical transition pathways. Research Policy, $\mathrm{n}^{\circ}$ 36, pp. 399-417.

Gerring J. (2004). What Is a Case Study and What Is It Good for? American Political Science Review, vol. 98, n 2, pp. 341-354.

Hinzen L., Vittori M., Etoa P., Chabrol D. (2010). Indications géographiques : défis et opportunités pour le secteur café-cacao au Cameroun. Yaoundé, CTA, p. 43.

Hütz-Adams F., Huber C., Knoke I., Morazan P., Mürlebach M. (2016). Renforcer la compétitivité de la production de cacao et augmenter le revenu des producteurs de cacao en Afrique de l'Ouest et en Afrique centrale. Bonn, Südwind Institut.

Huybrechts B. (2007). L'impact du commerce équitable sur les producteurs : les coopératives de café et de cacao en Bolivie (Mémoire de $D E A)$. Université de Liège, Liège.

IITA (2016). Farmer field school report. Yaoundé, International Institute of Tropical Agriculture, pp. 5-10.

Ingram J. (2018). Agricultural transition: Niche and regime knowledge systems' boundary dynamics. Environmental Innovation and Societal Transitions, $\mathrm{n}^{\circ}$ 26, pp. 117-135.

Ingram V., van Rijn F., Waarts Y., Gilhuis H. (2018). The impacts of cocoa sustainability initiatives in West Africa. Sustainability, vol. $10, \mathrm{n}^{\circ} 4249$.

Ingram V., Waarts Y., Ge L., van Vugt S., Wegner L., Puister-Jansen L., Tanoh F. (2014). Impact of UTZ certification of cocoa in Ivory Coast: Assessment framework and baseline. Wageningen, LEI Wageningen UR, 198 p.

KIT Royal Tropical Institute, Agroeco/Louis Bolk, and Tradin (2010). Organic cocoa 
production in Cameroon and Togo: feasibility study. Amsterdam.

Lemeilleur S., N'Dao Y., Ruf F. (2015). The productivist rationality behind a sustainable certification process: evidence from the Rainforest Alliance in the Ivorian cocoa sector. International Journal of Sustainable Development, vol. 18, $\mathrm{n}^{\circ}$ 4, pp. 310-328.

Long T. B., Blok V., Coninx I. (2016). Barriers to the adoption and diffusion of technological innovations for climate-smart agriculture in Europe: Evidence from the Netherlands, France, Switzerland and Italy. Journal of Cleaner Production, $\mathrm{n}^{\circ} 112$, pp. 9-21.

Mabah Tene G. L., Havard M., Temple L. (2013). Déterminants socio-économiques et institutionnels de l'adoption d'innovations techniques concernant la production de maïs à l'ouest du Cameroun. Tropicultura, vol. 31, n 2, pp. 137-142.

McDonald R., Heanue K., Pierce K., Horan B. (2016). Factors Influencing New Entrant Dairy Farmer's Decision-making Process around Technology Adoption. The Journal of Agricultural Education and Extension, vol. 22, $\mathrm{n}^{\circ}$ 2, pp. 163-177.

MINEPAT (2014). Rapport sur le développement économique du Cameroun : région du Centre Yaoundé, Cameroun, pp. 4-10.

Mithöfer D., Roshetko J. M., Donovan J. A., Ewane N., Robiglio V., Wau D., Blare T. (2017). Unpacking 'sustainable'cocoa: do sustainability standards, development projects and policies address producer concerns in Indonesia, Cameroon and Peru? International Journal of Biodiversity Science, Ecosystem Services \& Management, vol. 13, n 1, pp. 444-469.

Mwangi, M., Kariuki, S. (2015). Factors determining adoption of new agricultural technology by smallholder farmers in developing countries. Journal of Economics and sustainable development, vol. $6, \mathrm{n}^{\circ} 5$, pp. 208216.

N'Dao Y. (2012). Rationalités, changements de pratiques et impacts des standards durables sur les petits producteurs : le cas de la certification Rainforest Alliance dans le secteur du cacao. Mémoire de master 2, SupAgro Montpellier, Université de Montpellier 1.
Ngoucheme R., Kamdem C. B., Jagoret P., Havard M. (2016). Impact de la certification sur les performances agro-économiques des producteurs de cacao du centre Cameroun. In 2016 African Association of Agricultural Economists (AAAE) Fifth International Conference, September 23-26, Addis Ababa, Ethiopia, n 249294.

Ntsama E. S. M., Kamgnia D. B. (2008). Les déterminants de l'adoption des variétés améliorées de maïs : adoption et impact de $l a$ « CMS $8704 »$. CEA (Communauté économique africaine).

Ouattara S. (2015). Enjeux de la certification du cacao produit en Côte d'Ivoire. Revue canadienne de géographie tropicale, vol. $2, \mathrm{n}^{\circ} 2$, pp. 43-51.

Paustian M., Theuvsen L. (2017). Adoption of precision agriculture technologies by German crop farmers. Precision Agriculture, vol. 18, n 5, pp. 701-716.

Potts J., Lynch M., Wilkings A., Huppé G., Cunningham M., Voora V. (2014). The state of sustainability initiatives review 2014 : Standards and the green economy, pp. 14-45.

Ruf F., N'Dao Y., Lemeilleur S. (2013). Certification du cacao, stratégie à hauts risques. Inter-réseaux Développement rural, pp. 1-7.

Starr M. A. (2014). Qualitative and MixedMethods Research in Economics : Surprising Growth, Promising Future. Journal of Economic Surveys, vol. 28, $\mathrm{n}^{\circ} 2$, pp. 238-264.

Tankam C. (2017). L'organisation des marchés de producteurs de fruits et légumes biologiques à Nairobi, Kenya. Cahiers agricultures, vol. 26, n 35006.

Temple L., Mathe S., Machicou N. N., Fongang G., Ndoumbe N. M. (2017). Système national de recherche et d'innovation en Afrique: le cas du Cameroun. Innovations, art14_I-à art14_XXVI.

Temple L., Barret D., Blundo Canto G., Dabat, MH., Devaux-Spatarakis A., Faure G., Hainzelin E., Mathé S., Toillier A., Triomphe B. (2018). Assessing impacts of agricultural research for development: a systemic model focusing on outcomes. Research Evaluation, vol. 27, $\mathrm{n}^{\circ}$ 2, pp. 157-170. 
RECHERCHE

Anny Lucrèce NLEND NKOTT, Syndhia MATHÉ, Ludovic TEMPLE

UTZ (2015). Certification Protocol version 4.0. Consulté à l'adresse www.utzcertified.org.

Van Rijsbergen B., Elbers W., Ruben R., Njuguna S. N. (2016). The ambivalent impact of coffee certification on farmers' welfare: a matched panel approach for cooperatives in Central Kenya. World Development, $\mathrm{n}^{\circ}$ 77, pp. 277-292.
Vavra P. (2009). L'agriculture contractuelle: rôle, usage et raison d'être. Paris, OCDE.

Zeuli K. A., King R. P. (2004). The Impact of Organizational Form on Producer Contracting Decisions. Canadian Journal of Agricultural Economics, vol. 52, $\mathrm{n}^{\circ} 2$, pp. 147-164. 